\title{
Effects of lack of pulsatility on pulmonary endothelial function in the Fontan circulation
}

\author{
Roland Henaine, MD, ${ }^{a}$ Mathieu Vergnat, MD, ${ }^{a}$ Emile A. Bacha, MD, ${ }^{b}$ Bruno Baudet, BSc, ${ }^{c}$ \\ Virginie Lambert, $\mathrm{MD},{ }^{\mathrm{c}}$ Emre Belli, $\mathrm{MD},{ }^{\mathrm{c}}$ and Alain Serraf, $\mathrm{MD}, \mathrm{PhD}^{\mathrm{d}}$
}

\begin{abstract}
Objectives: Continuous flow in the Fontan circulation results in impairment of pulmonary artery endothelial function, increased pulmonary arterial resistance, and, potentially, late failure of Fontan circulation. We investigated the mechanisms of vascular remodeling and altered vascular reactivity associated with chronic privation of pulsatility on pulmonary vasculature.
\end{abstract}

\begin{abstract}
Methods: A total of 30 pigs were evenly distributed in 3 groups: 10 underwent a sham procedure (group I) and 20 underwent a cavopulmonary shunt between the superior vena cava and right pulmonary artery-10 with complete ligation of the proximal right pulmonary artery (group II, nonpulsatile) and 10 with partial ligation (group III, micropulsatile). At 3 months postoperatively, the in vivo hemodynamics, in vitro vasomotricity (concentration response curves on pulmonary artery isolated rings), and endothelial nitric oxide synthase protein level were assessed. A comparison between group and between the right and left lung in each group was performed.
\end{abstract}

Results: Group II developed right pulmonary hypertension and increased right pulmonary resistance. Endothelial function was altered in group II, as reflected by a decrease in the vasodilation response to acetylcholine and ionophoric calcium but preservation of the nonendothelial-dependent response to sodium nitroprusside. Group III micropulsatility attenuated pulmonary hypertension but did not prevent impairment of the endothelial-dependant relaxation response. Right lung Western blotting revealed decreased endothelial nitric oxide synthase in group II $(0.941 \pm 0.149$ vs sham $1.536 \pm 0.222, P=.045)$ that was preserved in group III $(1.275 \pm 0.236, P=.39)$.

Conclusions: In a chronic model of unilateral cavopulmonary shunt, pulsatility loss resulted in an altered endothelial-dependant vasorelaxation response of the pulmonary arteries. Micropulsatility limited the effects of pulsatility loss. These results are of importance for potential therapies against pulmonary hypertension in the nonpulsatile Fontan circulation, by retaining accessory pulmonary flow or pharmaceutical modulation of nonendothelial-dependant pulmonary vasorelaxation. (J Thorac Cardiovasc Surg 2013;146:522-9)

The Fontan operation ${ }^{1}$ has become the procedure of choice in the treatment of patients with a single anatomic or functional ventricle. The procedure places the systemic and pulmonary circulation in series, with the systemic circulation driven effectively by the single ventricle, and most of the pulmonary blood flow, which comes directly from the vena cava, driven by negative intrathoracic pressure and diastolic function of the single ventricle. It results in a pulmonary circulation very different from physiologic conditions, known as the "Fontan circulation."

\footnotetext{
From the Department of Cardiothoracic Surgery, ${ }^{a}$ Hôpital Louis Pradel, Hospices Civils de Lyon, Claude Bernard Lyon I University, Faculté de MédecineLaboratoire de Physiologie, Lyon, France; Department of Pediatric Cardiac

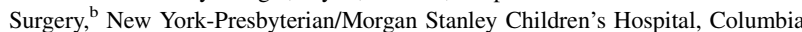
University, New York, NY; Laboratoire de Chirurgie Expérimentale, ${ }^{\mathrm{c}}$ Université Paris XI Hôpital Marie Lannelongue, Le Plessis-Robinson, France; and Department of Pediatric Cardiac Surgery, ${ }^{\mathrm{d}}$ Jacques Cartier Hospital, Massy, France.

Disclosures: Authors have nothing to disclose with regard to commercial support.

Received for publication Sept 20, 2012; accepted for publication Nov 9, 2012; available ahead of print Dec 10, 2012

Address for reprints: Roland Henaine, MD, Department of Cardiac Surgery C, Cardiologic Hospital Louis Pradel, Ave du Doyen Lépine, Lyon 69394 France (E-mail: roland.henaine@chu-lyon.fr).

0022-5223/\$36.00

Copyright (c) 2013 by The American Association for Thoracic Surgery http://dx.doi.org/10.1016/j.jtcvs.2012.11.031
}

The Fontan operation has been shown to benefit patients with a single functioning ventricle and low pulmonary resistance. ${ }^{2}$ Its outcome and the risk of Fontan failure are highly dependent on several risk factors such as ventricular function, pulmonary artery size and valve regurgitation; however, pulmonary vascular resistance (PVR) plays a key role. Even a slight increase in PVR can result in failure of the procedure. More and more evidence has shown that endothelial dysfunction (ie, the inability to react appropriately to various stimuli), is 1 of the root causes of Fontan failure in these patients. The Fontan physiology exerts a considerable effect on the pulmonary circulation, characterized by increased pulmonary vascular impedance. The lack of pulsatile blood flow has major effects on endothelial function, ${ }^{3}$ vascular recruitment, and lung vessel growth, which in turn influence PVR. ${ }^{4}$ The long-term consequences of these abnormalities are becoming of increasing concern as the population of survivors increases.

Several disadvantages regarding nonpulsatile pulmonary flow have been reported, in both global and microvascular pulmonary circulation..$^{5-7}$ The vasodilatory potential of pulsatile flow and wall shear stress are well recognized in both pulmonary ${ }^{8}$ and systemic vascular beds. ${ }^{9,10}$ Previous 

Abbreviations and Acronyms
$\mathrm{EC}_{50}=50 \%$ of the maximal response
eNOS $=$ endothelial nitric oxide synthase
Emax $=$ maximal response
$\mathrm{NO}=$ nitric oxide
PA = pulmonary artery
PAP = pulmonary artery pressure
PVP = pulmonary vein pressure
PVR = pulmonary vascular resistance
$\mathrm{SVC}=$ superior vena cava

experiments in acute animal models of nonpulsatile pulmonary circulation have revealed an increase in PVR.

Numerous experiments of the effect of pulsatility on the pulmonary endothelium have been performed in vitro, in nonphysiologic conditions, using an isolated lung preparation. The influence of the autonomous nervous system and other vasoactive hormonal agents was therefore excluded. In contrast, in vivo long-term experimental studies are not possible, because animal models of Fontan circulation do not exist.

Although the vascular morphology is well described, the mechanisms of vascular remodeling and altered vascular reactivity associated with chronic privation of pulsatility on pulmonary vasculature remain incompletely understood.

Despite the extensive analyses, the physiologic changes of pulmonary vasculature after cavopulmonary shunt have not been thoroughly investigated, and the underlying pathogenesis of steady flow-induced pulmonary hypertension of Fontan circulation remains poorly understood.

In the present study, we investigated the influence of flow pulsatility on the hemodynamics and vasomotricity of the pulmonary vasculature in a chronic porcine model of unilateral cavopulmonary shunt, using the contralateral lung as the control.

We hypothesized that preserving some antegrade pulsatility would maintain endothelial function and prevent steady flow-induced pulmonary hypertension.

Using in vivo hemodynamics measurements and in vitro vasoreactivity, we examined the effects of pulsatility, nonpulsatility, and micropulsatility blood flow on the pulmonary vascular bed.

We also determined the expression of endothelial nitric oxide (NO) synthase (eNOS) protein under these different pulsatility regimens.

\section{METHODS}

\section{Surgical Protocol}

Animal preparation. A total of 30 large white pigs weighing $20 \pm 2 \mathrm{~kg}$ were used in the present study. All the pigs received humane care in compliance with the "Principles of Laboratory Animal Care," formulated by the National Society for Medical Research, and the "Guide for the Care and Use of Laboratory Animals," published by the National Institutes of Health (NIH publication no. 85-23, revised 1996).

Anesthesia was induced with intramuscular ketamine $(10 \mathrm{mg} / \mathrm{kg})$ and atropine sulfate $(0.2 \mathrm{mg} / \mathrm{kg})$ and maintained with intravenous pentobarbital (10 mg/kg bolus; continuous infusion, $0.1 \mathrm{mg} / \mathrm{kg} / \mathrm{min}$ ).

The pigs were paralyzed with pancuronium $(0.3 \mathrm{mg} / \mathrm{kg})$. After endotracheal intubation, intermittent positive-pressure ventilation was provided (MMS RET 107 ventilator; MMS, Pau, France) at a tidal volume of $10 \mathrm{~mL} / \mathrm{kg}$, respiratory rate of 18 cycles $/ \mathrm{min}$, fraction of inspired oxygen of 0.5 . The body temperature was kept constant at $37^{\circ} \mathrm{C}$.

Surgical preparation. After midline sternotomy, the superior vena cava (SVC) was dissected, and the azygos and right mammary vein were ligated to allow full mobilization of the SVC. The right pulmonary artery (PA) was dissected, up to the superior upper lobe branch. The baseline hemodynamic values were recorded before additional manipulation: direct measurement of the aortic pressure, PA pressure (PAP; trunk, right branch), SVC pressure, and left and right pulmonary vein pressures (PVPs) was done; and the blood flow rate (with ultrasonic peritubular transit time flow probes and the T106/T206 Animal Research Flowmeter [Transonic System, Ithaca, NY]) of the aorta and PA trunk and PA right branch were measured. A dose of $100 \mathrm{IU} / \mathrm{kg}$ heparin was administered. For group I (sham group), no procedure was performed. In group II (nonpulsatile), the SVC was disconnected from the right atrium and anastomosed endto-side to the right PA, with ligation of the origin of the right PA. In group III (micropulsatile), a similar SVC-to-right PA anastomosis was performed, but the right PA was banded at its origin using a 3-mm Hegar dilator. The hemodynamic data were also collected after the procedure.

The total PVR ( $\mathrm{mm} \mathrm{Hg} / \mathrm{min} / \mathrm{L}$ ) was calculated by dividing the pressure decrease in $\mathrm{mm} \mathrm{Hg}$ between the PA and left atrium (Ppa - Pla) in $\mathrm{mm} \mathrm{Hg}$ by the flow $\mathrm{Q}$ in $\mathrm{L} / \mathrm{min}: \mathrm{PVR}=(\mathrm{Ppa}-\mathrm{Pla}) / \mathrm{Q}$.

To avoid postoperative occlusion of the anastomosis, the pigs were anticoagulated with fractionated heparin until the terminal study.

The terminal study was performed at 3 months. With the pigs under general anesthesia, the angiographic catheter was placed in the right PA through the right jugular vein, under fluoroscopic guidance. Correct patency of the anastomosis was controlled. Next, fourth intercostal space left thoracotomy was performed; the hemodynamic data were collected. Subsequently, right thoracotomy was performed, and the respective hemodynamic data were collected. After heparinization, the pigs were killed by exsanguination under deep anesthesia. The lungs were removed for histologic sampling.

\section{Histologic Examination}

The right and left inferior lobe fragments were harvested, and the tissues were fixed in $10 \%$ buffered formalin and imbedded in paraffin. Sections ( $4 \mu \mathrm{m}$ thick) were cut for light microscopic examination and stained with hematoxylin-phloxin-saffron and orcein-picroindigo-carmine.

\section{In Vitro Pulmonary Vasomotricity}

Four intrapulmonary arterial segments were isolated for each pig and each lung. Carefully preserving the integrity of endothelium and removing any adipose tissue, the vessels were cut into 3-mm-long and 4-mm external diameter rings. The samples were placed in tissue bath containing KrebsHenseleit bicarbonate buffer (composition $118.3 \mathrm{mM} \mathrm{NaCl}, 25 \mathrm{mM}$ $\mathrm{NaHCO}_{3}, 4.7 \mathrm{mM} \mathrm{KCl}, 1.2 \mathrm{mM} \mathrm{KH} \mathrm{PO}_{4}, 1.2 \mathrm{mM} \mathrm{MgSO}$, $2.5 \mathrm{mM}$ $\mathrm{CaCl}_{2}, 0.03 \mathrm{mM}$ ethylenediaminetetraacetic acid, and $11.1 \mathrm{mM}$ glucose) at $37^{\circ} \mathrm{C}$ and bubbled continuously with $95 \%$ oxygen and $5 \%$ carbon dioxide. The rings were then mounted onto stainless steel supports, suspended in 10-mL tissue baths, and connected to force displacement transducers (LB-5; Showa-Sokki, Tokyo, Japan) linked to an amplifier (emka Technologies, Falls Church, Va) and a computerized acquisition system to record any changes in force generated by the preparation. The resting tension was adjusted to $1 \mathrm{~g}$ and corresponded to the optimal length for tension development in the porcine PA. The rings were then equilibrated for 
60 to 90 minutes. Throughout the whole experiment, the tissue bath was replaced every 10 minutes.

After incubation with indomethacin $\left(10^{-5} \mathrm{M}\right)$ for 60 minutes, a concentration-response curve to phenylephrine $\left(10^{-9}-3 \cdot 10^{-4} \mathrm{M}\right)$ was obtained. The rings were then rinsed, and the developed force was allowed to return to baseline. The rings were then precontracted with phenylephrine to generate $1 \mathrm{~g}$ of developed force. Once a stable contraction was obtained, different pharmacologic protocols were performed.

Cumulative doses of acetylcholine $\left(10^{-9}-10^{-4} \mathrm{M}\right)$, calcium ionophore $\left(10^{-10}-3 \cdot 10^{-7} \mathrm{M}\right)$, sodium nitroprusside $\left(10^{-9}-10^{-4} \mathrm{M}\right)$ were added to the bath to assess the changes in endothelium-dependent relaxation. These rings were again washed and allowed to equilibrate to baseline levels.

The procedure was repeated with a single dose $\left(10^{-5} \mathrm{M}\right)$ of sodium nitroprusside, an endothelium-independent vasodilator.

In addition to the change in force, the responses were assessed by determining the concentration that produced $50 \%$ of the maximal response $\left(\mathrm{EC}_{50}\right)$, extrapolated from a plot of log concentration versus the percentage of maximal response.

The maximal response (Emax) represents the maximal intensity of the contraction or relaxation to an agonist. The contractile response to phenylephrine is expressed in absolute values ( $\mathrm{g}$ ), and the maximal relaxation to acetylcholine and calcium ionophore are expressed as percentages of the phenylephrine-induced precontraction, with $0 \%$ indicating no relaxation and $100 \%$ indicating a relaxation equal to that of the precontraction.

\section{Western Blot Analysis}

Four 2- to 3-g samples from each right lung were harvested, snap-frozen in liquid nitrogen, and stored at $-80^{\circ} \mathrm{C}$. The tissue was homogenized on ice using an Ultrathurax homogenizer (Kinematica, Lucerne, Switzerland) in $1 \mathrm{~mL}$ of CHAPS lysis buffer. The extracts were clarified by centrifugation ( $490 \mathrm{~g}$ for 15 minutes at $4^{\circ} \mathrm{C}$ ).

The supernatant was separated on $7 \%$ sodium dodecyl sulfatepolyacrilamide gel and electrophoretically transferred to polyvinylidene difluoride membranes (Immobilon transfer membrane, IPVH00010; EMD Millipore, Billerica, Mass). The membranes were blocked at room temperature with 5\% bovine serum albumin (A 4503-100G; Sigma Aldrich, St Louis, Mo) in Tris-buffered saline containing $0.1 \%$ Tween 20 . The membranes were incubated with mouse polyclonal anti-eNOS (1:1000, N30020; BD Transduction Laboratories, BD Biosciences, Franklin Lakes, NJ) diluted on blocking solution (1 hour at room temperature). The membranes were washed 2 times for 5 minutes and 1 time for 15 minutes with Tris-buffered saline containing $0.1 \%$ Tween 20 before they were hybridized with 1:10,000 dilution of anti-mouse horseradish peroxidase antibody (no. 401215, Calbiochem, EMD Millipore; 1 hour at room temperature). After the 2 times 5-minute and 1 time 15-minute washes, the bands were visualized with chemiluminescence on radiographic film (Kodak BioMax MS Film; Eastman Kodak Company, Rochester, NY) using an ECL Kit (Amersham, Arlington Heights, Ill).

A lung extract from a sham piglet was included on each gel as an internal control. Each membrane was probed for the protein of interest and then reprobed for $\beta$-actin. Each densitometric value (Gene Tools; Syngene, Cambridge, Mass) was divided by its $\beta$-actin control to obtain a relative value for the eNOS protein levels. The relative values were averaged and then corrected using a factor defined as the relative protein level of the internal control obtained from that particular blot divided by the relative protein level of the internal control from the reference membrane of the same protein of interest. The results are reported as the proportion of the internal control that equaled 1 .

\section{Statistical Analysis}

Data are expressed as the mean \pm standard deviation. Statistical analysis (for hemodynamic and vasoreactivity studies) was performed for comparison between groups at each point, between each point among same group, and, finally, between the right and left lung of the same pig (intraindividual control). Each pig served as its own control.

The data were analyzed with the software package, Statview IV (Abacus Concepts, Berkeley, Calif). Hemodynamic comparisons were performed using a paired Student $t$ test. The vasoreactivity comparisons were performed with 2-way analysis of variance followed by Fisher's exact test or a chi-square test for the between-group comparisons. The Western blot comparisons between groups were made using the unpaired Student $t$ test.

\section{RESULTS \\ Hemodynamic Data}

The baseline characteristics and hemodynamic data were similar between the groups (Table 1).

At 3 months, the patency of anastomosis was correct in all the pigs. The right PAP was significantly increased

TABLE 1. Hemodynamic, baseline, and terminal data in 3 groups

\begin{tabular}{|c|c|c|c|c|c|c|}
\hline \multirow[b]{2}{*}{ Variable } & \multicolumn{2}{|c|}{ Group I } & \multicolumn{2}{|c|}{ Group II } & \multicolumn{2}{|c|}{ Group III } \\
\hline & Baseline & $3 \mathrm{mo}$ & Baseline & $3 \mathrm{mo}$ & Baseline & 3 mo \\
\hline Pigs (n) & 10 & 10 & 10 & 10 & 10 & 10 \\
\hline Weight (kg) & $20 \pm 1$ & $40 \pm 8$ & $22 \pm 3$ & $54 \pm 7$ & $19 \pm 3$ & $46 \pm 3$ \\
\hline $\mathrm{AoP}(\mathrm{mm} \mathrm{Hg})$ & $52.8 \pm 12.4$ & $82.3 \pm 25.1$ & $56.6 \pm 7.4$ & $57.3 \pm 5.8$ & $54 \pm 3.1$ & $70 \pm 19.2$ \\
\hline $\operatorname{PAPr}(\mathrm{mm} \mathrm{Hg})$ & $9.0 \pm 2.8$ & $11.1 \pm 0.6$ & $9.6 \pm 3.8$ & $26.7 \pm 1.3^{*}, \dagger, \ddagger$ & $8.8 \pm 2.7$ & $14.2 \pm 0.8^{*}, \ddagger$ \\
\hline PAPl (mm Hg) & $10.9 \pm 1.8$ & $12.6 \pm 1.3$ & $10.4 \pm 1.9$ & $19.4 \pm 4.7 *, \ddagger$ & $11.8 \pm 1.3$ & $17.9 \pm 4.4^{*}, \ddagger$ \\
\hline PVPr (mm Hg) & $3.9 \pm 1.4 \S$ & $7.1 \pm 0.2$ & $4.6 \pm 1.3 \S$ & $14.1 \pm 3.0^{*}, \dagger, \ddagger$ & $3.8 \pm 2.2 \S$ & $7.4 \pm 2.6$ \\
\hline PVPl (mm Hg) & & $7.4 \pm 1.5$ & & $8.6 \pm 0.4 \ddagger$ & & $8.8 \pm 0.7$ \\
\hline Ao outflow & $1.90 \pm 0.13$ & $2.34 \pm 0.58$ & $2.10 \pm 0.47$ & $2.57 \pm 0.45$ & $2.00 \pm 0.22$ & $2.32 \pm 0.24$ \\
\hline SVC outflow & $0.55 \pm 0.06$ & NA & $0.80 \pm 0.36$ & NA & $0.52 \pm 0.21$ & NA \\
\hline PAr outflow & $1.27 \pm 0.05 \ddagger$ & $1.70 \pm 0.58 \ddagger$ & $1.32 \pm 0.27 \ddagger$ & $1.82 \pm 0.04 \ddagger$ & $1.30 \pm 0.15 \ddagger$ & $1.68 \pm 0.36 \ddagger$ \\
\hline PAl outflow & $0.69 \pm 0.03 \ddagger$ & $1.48 \pm 0.18 \ddagger$ & $0.71 \pm 0.14 \ddagger$ & $2.62 \pm 0.52^{*}, \dagger, \ddagger$ & $0.70 \pm 0.08 \ddagger$ & $2.16 \pm 0.37^{*}, \ddagger$ \\
\hline $\mathrm{PVR}$ right lung $(\mathrm{mm} \mathrm{Hg} / \mathrm{min} / \mathrm{L})$ & $5.47 \pm 1.82 \ddagger$ & $2.36 \pm 0.45$ & $4.69 \pm 2.13 \ddagger$ & $6.92 \pm 1.83^{*}, \dagger, \ddagger$ & $6.23 \pm 1.37 \ddagger$ & $4.20 \pm 1.36^{*}$ \\
\hline PVR left lung, $\mathrm{mm} \mathrm{Hg} / \mathrm{min} / \mathrm{L}$ & $10.16 \pm 3.20 \ddagger$ & $3.56 \pm 0.82$ & $8.70 \pm 3.73 \ddagger$ & $3.92 \pm 1.40 \ddagger$ & $11.58 \pm 2.40 \ddagger$ & $4.09 \pm 1.83$ \\
\hline
\end{tabular}

Data presented as mean \pm standard deviation. $A o P$, Mean aortic pressure; $P A l$, left pulmonary artery; $P A r$, right pulmonary artery; $P A P l$, mean left pulmonary arterial pressure; $P A P r$, mean right pulmonary arterial pressure; $P V P l$, mean left pulmonary vein pressure; $P V P r$, mean right pulmonary vein pressure; $P V R$, pulmonary vascular resistance; $A o$, aortic; $S V C$, superior vena cava; $N A$, not available. *Significant difference to group I. †Significant difference to group III. $\ddagger$ Significant difference to contralateral side. $§$ Pulmonary right and left vein pressure were identical and equal to left atrial pressure at baseline, with left atrial pressure presented. 
in both group II $(26.7 \pm 1.3 \mathrm{~mm} \mathrm{Hg}, P<.0001)$ and group III $(14.2 \pm 0.8 \mathrm{~mm} \mathrm{Hg}, P<.0001)$ compared with sham-operated controls (group I; $11.1 \pm 0.6 \mathrm{~mm}$ $\mathrm{Hg}$ ), with significant difference between groups II and III $(P=.0005)$. The right PVP $(14.1 \pm 3 \mathrm{~mm} \mathrm{Hg})$ was significantly increased in group II relative to groups I and III, but group III demonstrated no statistical variation compared with group I (sham). The PVR in the right lung was significantly greater in group II (6.92 \pm $1.83 \mathrm{~mm} \mathrm{Hg} / \mathrm{min} / \mathrm{L})$ than in group I $(2.36 \pm 0.45 \mathrm{~mm}$ $\mathrm{Hg} / \mathrm{min} / \mathrm{L} ; P=.001)$ or group III $(4.20 \pm 1.36 \mathrm{~mm}$ $\mathrm{Hg} / \mathrm{min} / \mathrm{L}, P=.001)$. The right PVR was significantly elevated in group III compared with group I (4.20 \pm 1.36 vs $2.36 \pm 0.45 \mathrm{~mm} \mathrm{Hg} / \mathrm{min} / \mathrm{L}, P=.001$ ) but to a lesser extent than in group II.

Finally, comparing the right and left lung of each pig, the PVR was greater in the right lung for group II $(P=.001)$ and was not statistically different from that of group I $(P=.12)$ or group III $(P=.88)$.

For the left lung, the left PAP was significantly increased in group II (19.4 $\pm 4.7 \mathrm{~mm} \mathrm{Hg}, P=.019)$ and group III $(17.9 \pm 4.4 \mathrm{~mm} \mathrm{Hg}, P=.05)$ relative to group I, but with no difference between groups II and III. No statistically significant difference was found among the 3 groups for PVP and PVR.

For the intraindividual comparison, no difference was found in sham group I, except for the PVR $(2.36 \pm 0.45$ for the right lung vs $3.56 \pm 0.82 \mathrm{~mm} \mathrm{Hg} / \mathrm{min} / \mathrm{L}$ for the left lung, $P=.001$ ). For group II, the right PAP was significantly greater than the left PAP $(26.7 \pm 1.3$ vs $19.4 \pm 4.7$ $\mathrm{mm} \mathrm{Hg}, P=.0003$ ), the right PVP was significantly greater than the left PVP $(14.1 \pm 3.0$ vs $8.6 \pm 0.4 \mathrm{~mm} \mathrm{Hg}$, $P=.0004)$, and the PVR was significantly greater in the right lung than in the left lung $(6.92 \pm 1.83$ vs $3.92 \pm 1.40 \mathrm{~mm} \mathrm{Hg} / \mathrm{min} / \mathrm{L}, P=.001)$.

For group III, no statistically significant increase was found between the right and left lung for PAP, PVP, or PVR. The PAP was even lower in the right lung (14.2 \pm 0.8 vs $17.9 \pm 4.4 \mathrm{~mm} \mathrm{Hg}, P=.003$ ).

\section{Histologic Findings}

No histologic changes were found in lung extract from the sham group I or group II and group III left lungs.

The right lung specimens from group II demonstrated alterations unlike those in groups I and III. Venous hyperplasia was noted, located along the bronchial axes, between the alveolar lobules, and under the pleura. The venous wall thickness was enlarged, with muscular hyperplasia of the media (Figure 1).

No histologic changes were found in the arterial walls.

\section{Vasoreactivity}

No difference in vasoreactivity was found between the right and left lungs in group I.

\section{Vasoconstrictor Response of Pulmonary Rings}

In group II (Figure 2, A), maximal tension response developed by phenylephrine was significantly greater in the right $\mathrm{PA}$ than in the left $\mathrm{PA}(3.75 \pm 0.37$ vs $1.95 \pm 0.35 \mathrm{mg}, P=.001)$, and the $\mathrm{EC}_{50}$ concentration was greater for the right PA than for the left PA (8.30 $10^{-7} \pm 1.910^{-7}$ vs $\left.5.610^{-7} \pm 1.810^{-7} \mathrm{M}, P=.323\right)$. In group III (Figure 2, $D$ ), no significant difference was found in the Emax or $\mathrm{EC}_{50}$ between the right and left PAs. A comparison of the right PAs from all 3 groups revealed no significant differences among the groups (Figure 3, A).

\section{Vasodilator Responses in Pulmonary Rings}

In the phenylephrine-preconstricted pulmonary rings, vasorelaxation was depressed in the group II right PA compared to the left PA, using acetylcholine (Emax, $49.9 \% \pm$ $7.2 \%$ vs $62.8 \% \pm 6.2 \%, P=.187 ; \mathrm{EC}_{50}, 1.910^{-7} \pm 5.0$ $10^{-8}$ vs $1.010^{-7} \pm 4.310^{-8} \mathrm{M}, P=.21$; Figure $2, B$ ) or calcium ionophore (Emax, $49.6 \% \pm 6.4 \%$ vs $75.3 \% \pm$ $6.2 \%, P=.07 ; \mathrm{EC}_{50}, 3.910^{-7} \pm 3.910^{-8}$ vs 3.3 $10^{-7} \pm 3.810^{-8} \mathrm{M}, P=.262$; Figure $\left.2, C\right)$, but without reaching statistical significance. In group III (Figure 2, $E, F)$, no significant difference was found in the Emax and $\mathrm{EC}_{50}$ between the right and left PAs.

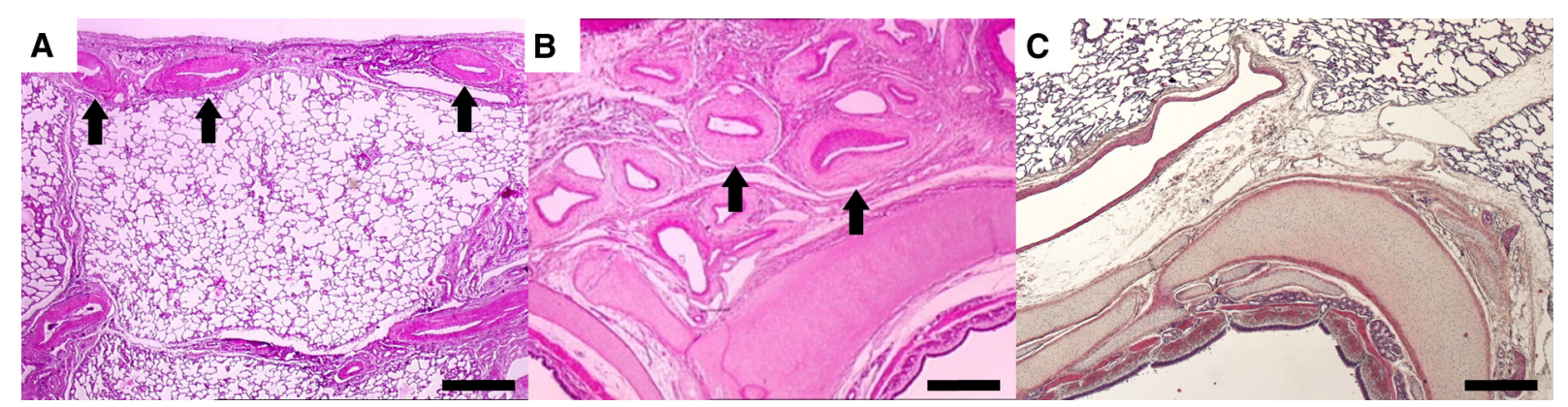

FIGURE 1. Example of lung sections ipsilateral to A, B, cavopulmonary anastomosis and C, control lung obtained from same pig at 3 months (representative of groups II and III) after cavopulmonary anastomosis, stained with hematoxylin-phloxin-saffron (magnification $\times 25$; scale bar, $500 \mu \mathrm{m}$ ). Arrowheads demonstrate hyperplasia of venous wall under pleura in Figure A, along bronchial axes and between alveolar lobules in Figure B of shunted lung. C, Control lung demonstrating normal histologic findings with normal venous wall thickness. 

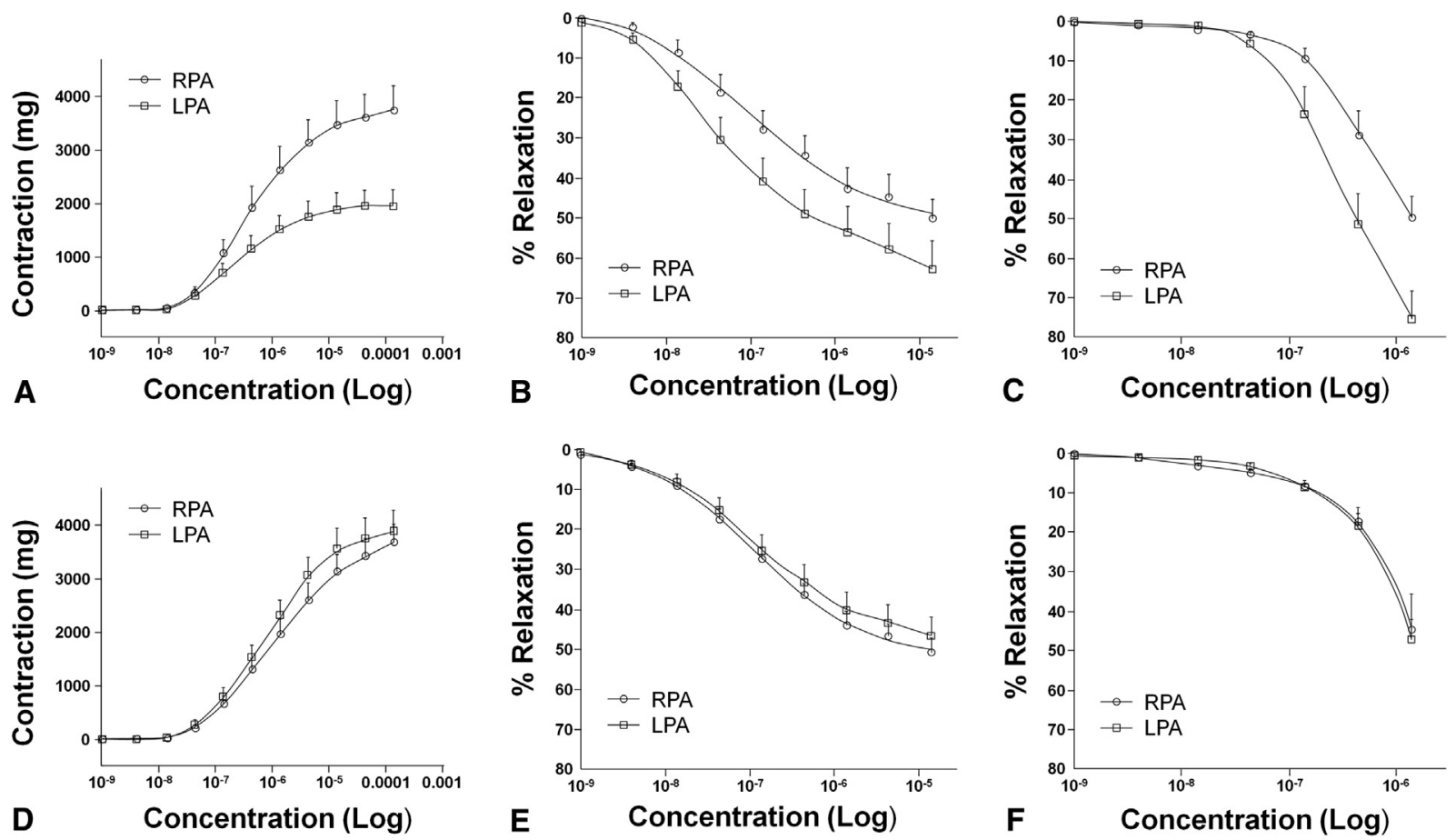

FIGURE 2. Dose-dependent responses of group II (nonpulsatile) right and left pulmonary artery (RPA and LPA, respectively) rings to cumulative addition of A, phenylephrine, B, acetylcholine, and C, calcium ionophore. Dose-dependent responses of group III (micropulsatile) RPA and LPA rings to cumulative addition of D, phenylephrine, E, acetylcholine, and F, calcium ionophore. A, D, Data presented as mean \pm standard deviation of developed tension in grams for contraction. B, C, E, F, Data presented as mean \pm standard deviation of percentage of dilatation relative to phenylephrine-induced preconstriction level for relaxation. RPA of group II demonstrated significantly different contraction and reduced relaxation (nonsignificant) relative to LPA. A, Phenylephrineinduced $\left(10^{-9}-3.10^{-4} \mathrm{M}\right)$ contraction in PA rings. Two-way analysis of variance revealed that RPA rings exhibited greater contraction to phenylephrine than LPA rings (Emax, $\left.P=.001 ; \mathrm{EC}_{50}, P=.323\right)$. B, Acetylcholine-induced $\left(10^{-9}-10^{-4} \mathrm{M}\right)$ relaxation in phenylephrine-preconstricted PA rings. Despite reduced RPA ring relaxation, no significant difference was found (Emax, $\left.P=.187 ; \mathrm{EC}_{50}, P=.210\right)$. C, Calcium ionophore-induced $\left(10^{-10}-3.10^{-7} \mathrm{M}\right)$ relaxation in phenylephrine-preconstricted PA rings. Two-way analysis of variance revealed that RPA rings exhibited blunted relaxation responses to calcium ionophore compared with responses from left lung (Emax, $P=.07 ; \mathrm{EC}_{50}, P=.262$ ). RPA of group III demonstrated restored similar level of contraction and relaxation, relative to LPA. D, Phenylephrine-induced $\left(10^{-9}-3.10^{-4} \mathrm{M}\right)$ contraction in PA rings. Two-way analysis of variance revealed that restored pulsatility abolished abnormal right pulmonary response with no difference in contraction to phenylephrine between RPA and LPA rings (Emax, $P=.715$; $\left.\mathrm{EC}_{50}, P=.08\right)$. E, Acetylcholine-induced $\left(10^{-9}-10^{-4} \mathrm{M}\right)$ relaxation in phenylephrine-preconstricted PA rings. Two-way analysis of variance revealed that restored pulsatility abolished abnormal right pulmonary response, with no difference in relaxation to acetylcholine between RPA and LPA rings (Emax, $\left.P=.495 ; \mathrm{EC}_{50}, P=.233\right)$. F, Calcium ionophore-induced $\left(10^{-10}-3.10^{-7} \mathrm{M}\right)$ relaxation in phenylephrine-preconstricted PA rings. Two-way analysis of variance revealed that restored pulsatility abolished abnormal right pulmonary response, with no difference in relaxation to calcium ionophore between RPA and LPA rings (Emax, $P=.796 ; \mathrm{EC}_{50}, P=.879$ ).

No difference was demonstrated in vasorelaxation to nitroprusside between the PAs (Emax in left and right PAs, group I, $115 \% \pm 19 \%$ vs $113 \% \pm 19 \%$; group II, $108 \% \pm 18 \%$ vs $115 \% \pm 18 \%$; group III, $97 \% \pm 11 \%$ vs $101 \% \pm 11 \%$, respectively).

\section{Interindividual Comparison of Vasoreactivity}

A comparison of the right PAs from groups I, II, and III revealed no significant differences among the groups with acetylcholine (Figure 3, $B$ ) but significantly depressed vasorelaxation (Figure 3, $C$ ) was noted in group II (Emax, $49.6 \% \pm 6.4 \%, P=.0003 ; \mathrm{EC}_{50}, 3.910^{-7} \pm 3.910^{-8}$ $\mathrm{M}, P=.001)$ and group III (Emax, $44.6 \% \pm 7.3 \%$, $\left.P=.002 ; \mathrm{EC}_{50}, 4.210^{-7} \pm 4.310^{-8} \mathrm{M}, P=.003\right)$ right PAs with calcium ionophore compared with group I
(Emax, 76.8\% $\left.\pm 6.7 \% ; \mathrm{EC}_{50}, 2.410^{-7} \pm 2.910^{-8} \mathrm{M}\right)$. No difference was demonstrated between group II and III right PA vasorelaxation to calcium ionophore.

\section{Immunoblot Analysis}

Immunoblot analyses of lung homogenates revealed that eNOS protein content was different among the groups (Figure 4). eNOS protein content was significantly lower in group II than in group I $(0.941 \pm 0.149$ vs $1.536 \pm$ $0.222, P=.045)$. However, group III demonstrated no significant decrease in eNOS content $(1.275 \pm 0.236, P=.39)$.

\section{DISCUSSION}

The results from the present study have indicate that, in a chronic porcine model of unilateral cavopulmonary shunt, 


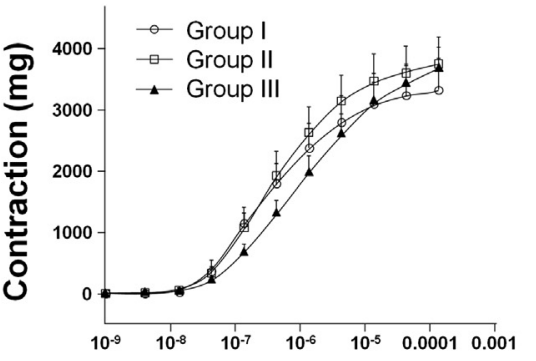

A

Concentration ( $\log )$
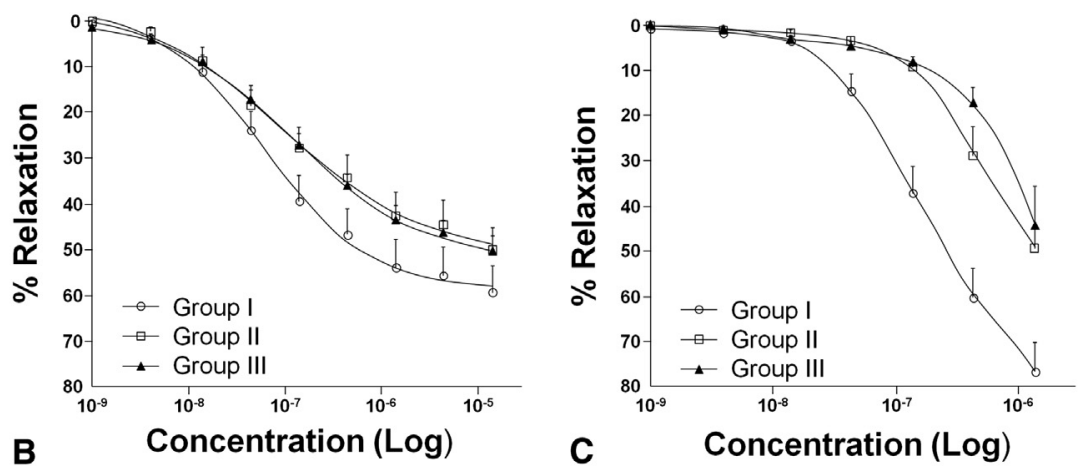

FIGURE 3. Dose-dependent responses of group I (sham), group II (nonpulsatile), and group III (micropulsatile) right pulmonary artery ( $P A$ ) rings to cumulative addition of phenylephrine, acetylcholine, calcium ionophore, and nitroprusside. In right PA, no difference in contraction was found among 3 groups, but significantly reduced dilatation response was found in groups II and III, relative to sham group I. A, Phenylephrine-induced $\left(10^{-9}-3.10^{-4} \mathrm{M}\right)$ contraction in PA rings. Two-way analysis of variance revealed contractile responses of right PA rings were similar among groups. $\mathrm{B}$, Acetylcholine-induced $\left(10^{-9}-10^{-4} \mathrm{M}\right)$ relaxation in phenylephrine-preconstricted PA rings. Despite reduced relaxation in groups II and III compared with group I, no significant difference was found (groups II and III compared with group I, Emax, $P=.333$ and $P=.334$, respectively). C, Calcium ionophore-induced $\left(10^{-10}-3.10^{-7} \mathrm{M}\right)$ relaxation in phenylephrine-preconstricted PA rings. Two-way analysis of variance revealed that group II and III right PA rings exhibited blunted relaxation responses to calcium ionophore compared with responses of sham group I (groups II and III compared with group I, Emax, $P=.002$ and $P=.0003, \mathrm{EC}_{50}, P=.003$ and $P=.001$, respectively). No significant difference was found in relaxation responses between groups II and III.

the lack of pulsatility results in unilateral elevation of PAP and PVR. Nonpulsatile flow decreased the endothelialdependent vasorelaxation response of the PAs. Finally, expression of eNOS was depressed in the nonpulsatile circulation lungs.

To the best of our knowledge, we are the first to study, in a chronic animal model, the hemodynamics and vasomotor function of the pulmonary vasculature exposed to nonpulsatile blood flow. The right pulmonary endothelium is subjected to continuous flow and the left lung receives pulsatile flow under physiologic conditions. Thus, each pig could act as its own control.

The hemodynamic consequence of nonpulsatile flow and thus the loss of shear stress in the right lung of group II (nonpulsatile) was a significant increase in right PAP compared with that of the left lung. The PVR followed the same course. For group III, partial antegrade pulsatile flow resulted in limitation of pulmonary hypertension, because the right PAP, even if significantly greater than that in the sham group, was significantly lower than that in group II and also lower than the left PAP in group III.

From a functional standpoint, pulsatility modulation also resulted in significant changes in pulmonary vasoreactivity. In the present study, we, for the first time, have characterized the vasoreactivity consequences of in vivo chronic pulsatility loss on pulmonary vasculature. In group II, the loss of pulsatility resulted in a significant decrease in the vasodilator response to acetylcholine and calcium inophore. In group III, antegrade pulsatile flow abolished the differences between the right and left lung vasodilator response. However, when considering the vasodilator response of the right lung of the 3 groups, both groups II and III demonstrated an impaired response compared with sham group I, reflecting the loss of pulsatility in group II and, to a lower extent, in group III. Numerous experimental studies ${ }^{11,12}$ using $\mathrm{NO}$ antagonists ( $\mathrm{N}$-methyl-L-arginine or methylene blue) demonstrated that the response to acetylcholine was endothelial dependent and used NO as the mediator. The findings were also corroborated in clinical studies. ${ }^{13,14}$ Pulsatility influences the hemodynamics by acting on endothelial function. In a clinical report on patients with a bidirectional cavopulmonary shunt, acetylcholine-induced vasorelaxation (endothelium-dependent vasorelaxation) was significantly attenuated, but nitroglycerin (exogenous NO donor and, thus, endothelium-nondependent vasorelaxation) was well preserved. ${ }^{3}$ Our data have corroborated these findings, because acetylcholine relaxation of the right lung was altered, but the nitroprusside response was preserved. These results suggest that Fontan circulation, by the loss of pulsatility, is responsible for endothelial dysfunction, and the PA smooth muscle relaxation response is maintained. The precise mechanism of acetylcholine response attenuation remains to be explored. In vitro studies have demonstrated that shear stress loss resulted in lower transcription of fibroblast and platelet-derived growth factors ${ }^{15}$ and of eNOS. ${ }^{16}$ Flow pulsatility has been demonstrated to be a powerful stimulus for NO release. ${ }^{17}$

In contrast to the acetylcholine and calcium inophore response, the vasodilator response to nitroprusside was preserved in all groups and on both sides in each group. Nonendothelium-dependent vasorelaxation was not altered by the cavopulmonary bypass procedure, regardless of group or lung side. One of the main contributors to late failure of the Fontan circulation is the increase in PVR. 

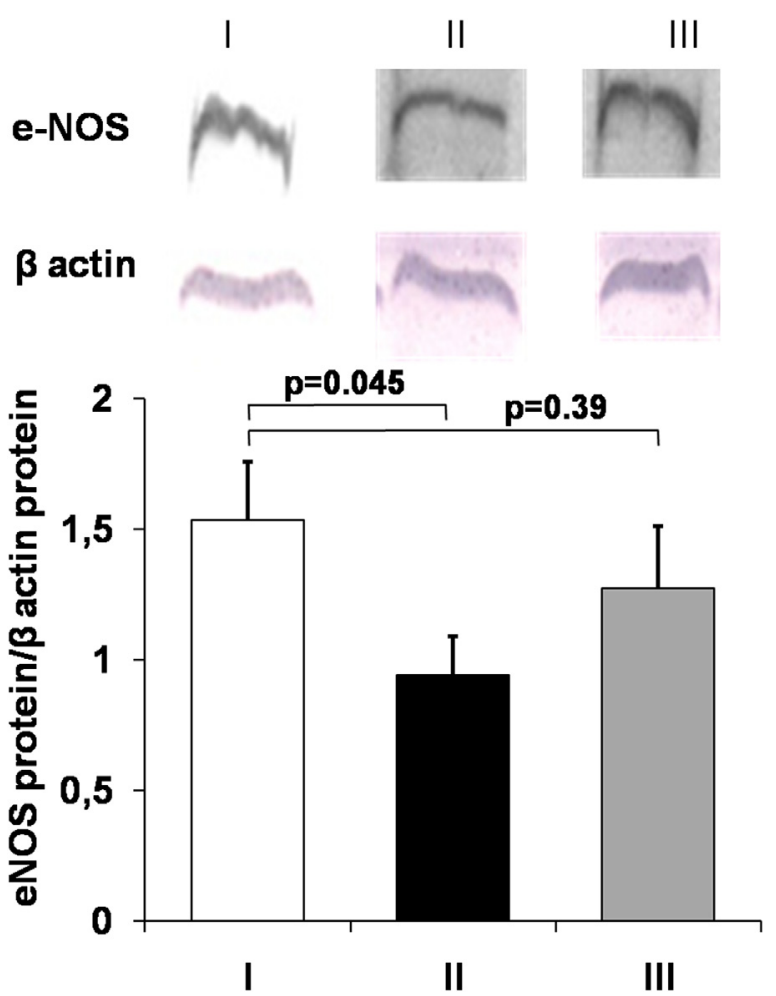

FIGURE 4. Immunoblot analysis for endothelial nitric oxide synthase (eNOS) protein in lung tissue from 3 groups. Top, Representative immunoblot from protein extracts prepared from lung tissue of pig from indicated group separated on $7.5 \%$ sodium dodecyl sulfate-polyacrylamide gel, electrophoretically transferred to Hybond membranes, and analyzed using a specific antiserum raised against eNOS. Bottom, Summary data from $10 \mathrm{pigs} / g r o u p ;$ densitometric values for eNOS protein normalized to $\beta$-actin protein for eNOS protein $/ \beta$-actin protein. Data presented as mean \pm standard deviation, normalized. eNOS protein $/ \beta$-actin protein decreased by $39 \%$ in nonpulsatile (group II, $P=.045$ ) and $17 \%$ in micropulsatility (group III, $P=.39$ ).

Because of the maintained response to nitroprusside in nonpulsatile or low-pulsatility circulations, the use of exogenous NO agents might impede the long-term PVR increase in Fontan patients.

The restoration of partial pulmonary pulsatility in group III, to prevent the effects of Fontan circulation, resulted in limitation of pulmonary hypertension. These results suggest that some residual antegrade pulsatile flow might play a protective role on nonpulsatile flow-induced endothelial dysfunction. However, such a protective role was not verified in our vasomotricity study. In the clinical field, despite initially controversial, the utility of a maintained residual antegrade flow, after cavopulmonary shunt, has been advocated by some investigators. ${ }^{18-20}$

To further determine the molecular mechanisms underlying endothelial functional abnormalities, we investigated the protein expression of eNOS in the remodeled vascular wall.
Patients with failing Fontan circulation have been shown to overexpress eNOS relative to normal controls and patients with good surgical outcomes, possibly related to endothelial dysfunction. ${ }^{21}$ However, in these patients, increased shear stress is caused by abnormal pulmonary blood flow, and polycythemia might cause endothelial dysfunction, with overstimulation of the NO pathway to maintain low pulmonary arterial pressure. eNOS is upregulated in shunt-induced pulmonary hypertension in lambs, in rats exposed to hypoxia, and in polycythemic rats. ${ }^{22-24}$ In contrast, in adult patients with pulmonary hypertension, eNOS expression is reduced. $^{25}$

We found that protein expression of eNOS was significantly decreased in the nonpulsatile right lung of group II and restored in the micropulsatile group III. This finding is in line with our observation that endothelial-dependent vasorelaxation is altered in group II, indicating that pulmonary hypertension is likely due to endothelial dysfunction. Because NO produces vasodilation and inhibits smooth muscle cell mitogenesis, restored NO production, which was induced by micropulsatility, might represent a therapeutic response to minimize the PVR in the setting of Fontan circulation.

\section{CONCLUSIONS}

The results of the present study have indicated that, in a chronic porcine model of unilateral cavopulmonary shunt, the lack of pulsatility results in unilateral elevation of PAP and PVR. Nonpulsatile flow decreased the endothelialdependent vasorelaxation response of PAs. Using Western blot analysis, we found that eNOS expression was depressed in the nonpulsatile circulation lungs.

Because nonendothelial-dependent vasorelaxation was maintained, endothelial dysfunction could be counterbalanced. Therefore, nonendothelial-dependent relaxing agents might represent a potential therapy for failing Fontan circulation with an elevated PAP. Finally, because pulsatility is an important modulator of pulmonary vascular tone, we demonstrated that micropulsatility limited the effects of pulsatility loss. Our observation supports the importance of maintaining accessory pulmonary blood flow in patients with a bidirectional cavopulmonary shunt, from the standpoint of pulmonary endothelial function.

We thank Dr Pascal Chiari for his critical appraisal of our report and constructive comments.

\section{References}

1. de Leval MR. The Fontan circulation: a challenge to William Harvey? Nat Clin Pract Cardiovasc Med. 2005;2:202-8.

2. Mair DD, Puga FJ, Danielson GK. The Fontan procedure for tricuspid atresia: early and late results of a 25 -year experience with 216 patients. J Am Coll Cardiol. 2001;37:933-9.

3. Kurotobi S, Sano T, Kogaki S, Matsushita T, Miwatani T, Takeuchi M, et al. Bidirectional cavopulmonary shunt with right ventricular outflow patency: the 
impact of pulsatility on pulmonary endothelial function. $J$ Thorac Cardiovasc Surg. 2001;121:1161-8.

4. Khambadkone S, Li J, de Leval MR, Cullen S, Deanfield JE, Redington AN. Basal pulmonary vascular resistance and nitric oxide responsiveness late after Fontan-type operation. Circulation. 2003;107:3204-8.

5. Furuse A, Brawley RK, Gott VL. Pulsatile cavopulmonary artery shunt: surgical technique and hemodynamic characteristics. J Thorac Cardiovasc Surg. 1972; 63:495-500.

6. Raj JU, Kaapa P, Anderson J. Effect of pulsatile flow on microvascular resistance in adult rabbit lung. J Appl Physiol. 1992;72:73-81.

7. Taguchi S, Yozu R, Iseki H, Soma Y, Inoue T. Effects of nonpulsatile and pulsatile right ventricular bypass on pulmonary circulation. Trans Am Soc Artif Intern Organs. 1988;34:213-21.

8. Saito O, Lamm WJ, Hildebrandt J, Albert RK. Pulsatile and nonpulsatile pressure-flow relationships in zone 3 excised rabbit lungs. J Appl Physiol. 1994;76:370-9.

9. Hutcheson IR, Griffith TM. Release of endothelium-derived relaxing factor is modulated both by frequency and amplitude of pulsatile flow. Am J Physiol Heart Circ Physiol. 1991;261:H257-62.

10. Pohl U, Busse R, Kuon E, Bassenge E. Pulsatile perfusion stimulates the release of endothelial autacoids. J Appl Cardiol. 1986;1:215-35.

11. Cooke JP, Stamler J, Andon N, Davies PF, McKinley G, Loscalzo J. Flow stimulates endothelial cells to release a nitrovasodilator that is potentiated by reduced thiol. Am J Physiol Heart Circ Physiol. 1990;259:H804-12.

12. Fineman JR, Crowley MR, Heymann MA, Soifer SJ. In vivo attenuation of endothelium-dependent pulmonary vasodilatation by methylene blue. $J$ Appl Physiol. 1991;71:735-41.

13. Cooper CJ, Landzberg MJ, Anderson TJ, Charbonneau F, Creager MA, Ganz P, et al. Role of nitric oxide in the local regulation of pulmonary vascular resistance in humans. Circulation. 1996;93:266-71.

14. Katz SD, Scharwz M, Yuen J, LeJemtel TH. Impaired acetylcholine-mediated vasodilation in patients with congestive heart failure: role of endothelium-derived vasodilating and vasoconstricting factors. Circulation. 1993;88:55-61.
15. Malek AM, Gibbons GH, Dzau VJ, Izumo S. Fluid shear stress differentially modulates expression of genes encoding basic fibroblast growth factor and platelet-derived growth factor B chain in vascular endothelium. J Clin Invest. 1993;92:2013-21.

16. Norris M, Morigi M, Donadelli R, Aiello S, Foppolo M, Todeschini M, et al. Nitric oxide synthesis by cultured endothelial cells are modulated by flow conditions. Circ Res. 1995;76:536-43.

17. Rubanyi GM, Romero JC, Vanhoutte PM. Flow induced release of endotheliumderived relaxing factor. Am J Physiol Heart Circ Physiol. 1986;250:H1145-9.

18. Berdat PA, Belli E, Lacour-Gayet F, Planche C, Serraf A. Additional pulmonary blood flow has no adverse effect on outcome after bidirectional cavopulmonary anastomosis. Ann Thorac Surg. 2005;79:29-36.

19. Gérelli S, Boulitrop C, Van Steenberghe M, Maldonado D, Bojan M, Raisky O, et al. Bidirectional cavopulmonary shunt with additional pulmonary blood flow: a failed or successful strategy? Eur J Cardiothorac Surg. 2012;42:513-9.

20. Turner ME, Richmond ME, Quaegebeur JM, Shah A, Chen JM, Bacha EA, et al. Intact right ventricle-pulmonary artery shunt after stage II palliation in hypoplastic left heart syndrome improves pulmonary artery growth. Pediatr Cardiol Epub 2012 Nov 15.

21. Lévy M, Danel C, Laval AM, Leca F, Vouhe PR, Israël-Biet D. Nitric oxide synthase expression by pulmonary arteries: a predictive marker of Fontan procedure outcome? Thorac Cardiovasc Surg. 2003;125:1083-90.

22. Black SM, Fineman JR, Steinhorn RH, Bristow J, Soifer SJ. Increased endothelial NOS in lambs with increased blood flow and pulmonary hypertension. Am J Physiol Heart Circ Physiol. 1998;275:H1643-51.

23. Resta TC, Chicoine LG, Omdahl JL, Walker BR. Maintained upregulation of pulmonary e-NOS gene and protein expression during recovery from chronic hypoxia. Am J Physiol Heart Circ Physiol. 1999;276:H699-708.

24. Walker BR, Resta TC, Nelin LD. Nitric oxide-dependent pulmonary vasodilation in polycythemic rats. Am J Physiol Heart Circ Physiol. 2000;279:H2382-9.

25. Giaid A, Saleh D. Reduced expression of endothelial nitric oxide synthase in the lungs of patients with pulmonary hypertension. $N$ Engl J Med. 1995;333: 214-24. 